\title{
因HAD
}

DOI: http://doi.org/10.22585/hospdomic.v6i1.150

\section{Lidocaína endovenosa domiciliaria en cuidados paliativos}

\section{Domiciliary endovenous lidocaine treatment in palliative care patients}

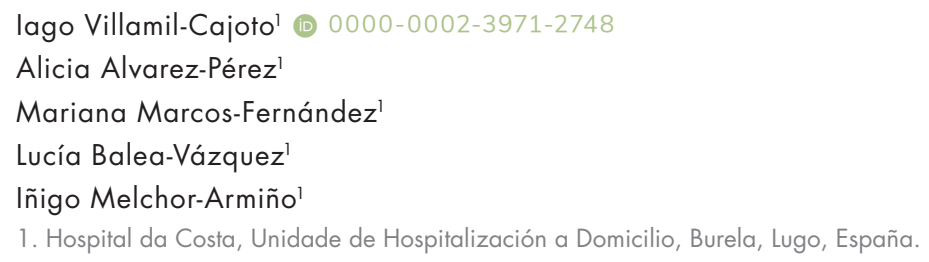

\section{Correspondencia/Correspondence}

lago Villamil Cajoto; iago.villamil.cajoto@sergas.es

Unidad Hospitalización a Domicilio- Cuidados paliativos.

Recibido/Received

12.12.2021

Aceptado/Accepted

13.12.2021
Conflicto de Intereses/Competing interest

No existe ningún tipo de conflicto de interés.

Financiación/Funding

Este trabajo no ha tenido ningún tipo de financiación.

Contribuciones de autoría/Author contributions

Los autores y autoras de este trabajo han contribuido por igual. 


\section{RESUMEN}

Introducción: El dolor neuropático es, en numerosas ocasiones, un dolor de difícil manejo que precisa la combinación de diferentes grupos terapéuticos para su control óptimo.

Método: La lidocaína es un anestésico local y su uso endovenoso es necesario ocasionalmente para el tratamiento del dolor neuropático; no hay experiencia en su uso domiciliario.

Resultados: Realizamos la infusión endovenosa domiciliaria con lidocaína en 2 pacientes con dolor neuropático refractario con mejoría clínica.

Conclusiones: La infusión de lidocaína endovenosa en domicilio es una técnica segura y eficaz en pacientes con dolor neuropático.

Palabras clave: Servicios de Atención de Salud a Domicilio; Lidocaína; Cuidados Paliativos; DoIor; Dimensión del Dolor.

\section{ABSTRACT}

Introduction: Neuropathic pain is a often difficult to control pain and is suitable of different therapeutic approaches.

Method: Lidocaine is a local anesthesic and its endovenous use is sometimes necessary to control neuropathic pain. There is no reported use of this drug at home.

Results: We performed the lidocaine endovenous infusion in two patients with neuropathic pain achieving significant clinical improvement.

Conclusions: Endovenous lidocaine infusion at home, is a safe and effective proceeding for patients with neuropathic pain.

Keywords: Home Care Services; Lidocaine; Palliative Care; Pain; Pain Measurement. 


\section{INTRODUCCIÓN}

En el paciente oncológico en cuidados paliativos, el dolor es un síntoma muy frecuente(1). El dolor neuropático (DNP) es en numerosas ocasiones de difícil control y precisa la combinación de diferentes grupos terapéuticos. Los pacientes oncológicos pueden desarrollar DNP por distintas causas: compresión o infiltración directa de estructuras nerviosas, traumatismos directos nerviosos, como consecuencia de procedimientos diagnósticos o quirúrgicos, y lesiones nerviosas secundarias a tratamientos como la radioterapia o la quimioterapia (neuropatía inducida por quimioterapia). Su presencia interfiere en la calidad de vida de los pacientes ${ }^{(2)}$.

La correcta aplicación de la escala analgésica de la OMS hasta su tercer escalón permite controlar el dolor en numerosos casos, pero no en todos. Entre los diferentes tratamientos precisos para el control del DNP, desde hace años y tras su experiencia en el control del dolor postoperatorio(3), se usa la lidocaína endovenosa.

La lidocaína, un anestésico local tipo amida actúa bloqueando los canales de sodio dependientes de voltaje en tejidos neuronales, interrumpiendo la transmisión nerviosa ${ }^{(4)}$. Su uso endovenoso lo hace casi exclusivo hospitalario, aunque no existe limitación para uso extrahospitalario siempre que se realice en condiciones de seguridad. Presentamos la experiencia de nuestra unidad de Hospitalización a Domicilio $(\mathrm{HaD})$ de lidocaína endovenosa en el domicilio de dos pacientes a tratamiento paliativo con DNP.

\section{DESARROLLO DE LA EXPERIENCIA}

Nuestra unidad pertenece a un Hospital Comarcal para un área sanitaria de 75000 pacientes. Durante los meses de agosto a diciembre de 2020, tratamos a 2 pacientes de 57 y 58 años con lidocaína endovenosa en domicilio para control de DNP.

De acuerdo con la unidad de dolor del servicio de Anestesia y Reanimación, protocolizamos la infusión de lidocaína endovenosa domiciliaria. Los pacientes ya habían recibido previamente lidocaína endovenosa sin incidencias en el ámbito hospitalario. Ambos pacientes disponían de reservorio endovenoso. Su ECG basal no presentaba patología. Se disponía durante el procedimiento, de expansores de volumen y del equipo estándar de reanimación avanzada disponible en HaD. Se realiza monitorización continua de TA, saturación y frecuencia cardíaca con equipo médico de facultativo y enfermería. Los pacientes firmaron un consentimiento informado para la administración de la medicación.

Se utilizaron viales de $50 \mathrm{mg} / \mathrm{ml}$ de lidocaína en 250 cc de glucosado $5 \%$, pasando en 40 minutos a dosis estimadas de $3 \mathrm{mg} / \mathrm{kg}$.

\section{Paciente 1}

Varón de 58 años con diagnóstico 5 años antes de adenocarcinoma moderadamente diferenciado de pulmón estadio IV con masa lobulada, basal posterior lóbulo inferior izquierdo, que contacta con la pleura a nivel posterior, y afección del músculo escaleno anterior izquierdo. Presenta impotencia funcional (antiálgica) e hiperestesia de características neuropáticas en la extremidad superior izquierda.

Recibe analgesia con $125 \mu \mathrm{g}$ de fentanilo transdérmico, rescates transmucosos de $200 \mu \mathrm{g}$, pregabalina $75 \mathrm{mg} / 12$ horas. así como dexametasona $2 \mathrm{mg} / 24$ horas, dexketoprofeno $25 \mathrm{mg} / 12$ horas 
y ocasionalmente metamizol (550 mg). Su situación basal: Escala Visual Analógica (EVA) 5 con picos de 8-9.

Se planifican perfusiones de lidocaína intravenosa (IV), 10 sesiones con el siguiente esquema en dosis ascendentes: $1^{\circ}$ día: $100 \mathrm{mg}$ lidocaína; $2^{\circ}$ día: $100 \mathrm{mg} ; 3^{\circ}$ día: $150 \mathrm{mg}, 4^{\circ}$ día: $150 \mathrm{mg} ; 5^{\circ}$ día: 150 mg; $6^{\circ}$ día: 200 mg; $7^{\circ}$ día: 200 mg; 8ª día 250 mg; 9 día: 300 mg; $10^{\circ}$ día: 300 mg; dosis final de $3 \mathrm{mg} / \mathrm{kg}$.

Se realiza la administración sin incidencias en ninguna de las sesiones de tratamiento. Durante el tratamiento, presenta una mejoría clínica evidente pasando a un EVA basal 2-3 con picos mucho menos frecuentes y considerados 5-7 por el paciente. En el final de tratamiento EVA basal 2-3, picos de 5-6 y destaca mejoría en la respuesta a rescates con fentanilo transmucoso.

\section{Paciente 2}

Mujer de 57 a con diagnóstico 9 años antes de timoma mediastínico con progresión tras varias cirugías y líneas de citostático, en este momento irresecable. Presenta polineuropatía paraneoplásica de características neuropáticas en extremidades inferiores por la que recibe 2 ciclos de inmunoglobulinas endovenosas con mejoría limitada, se encuentra a tratamiento con fentanilo transdérmico a dosis de $150 \mu \mathrm{g}$, rescates transmucosos de $200 \mu \mathrm{g}$, gabapentina $300 \mathrm{mg} / 8$ horas y dexametasona $2 \mathrm{mg} / 24$ horas. Persisten las molestias en relación con polineuropatía sensitiva dolorosa subaguda paraneoplásica.

Se realiza tratamiento endovenoso de 10 ciclos a dosis completas con lidocaína 300 mg (3mg/ $\mathrm{kg})$.

La situación basal con respecto al dolor era un EVA 2-3 con picos de EVA 9. Durante la infusión se consigue control absoluto de molestias. La situación basal tras el ciclo de 10 días de lidocaína pasa a EVA 2 picos aislados de 5-6. Presenta así mismo una mejoría notable de la percepción de calidad de vida (aunque no medida con instrumento-escala) al mejorar la deambulación, afectada por el dolor previo.

\section{CONCLUSIONES}

El dolor refractario al uso de opiáceos, especialmente el DNP, es un desafío para los sanitarios a cargo de estos pacientes. El efecto analgésico de la lidocaína por vía endovenosa está comprobado desde hace años y se consigue al interrumpir la transmisión nerviosa de las descargas ectópicas provocadas en un neuroma ${ }^{(5)}$. Podría tener así mismo un efecto central, a nivel espinal a través del enlace con el receptor del N-metil-D aspartato( ${ }^{(6)}$. Hay evidencia de seguridad en su uso controlado con algún reciente meta-análisis que resume los escasos ensayos clínicos disponibles ${ }^{(7)}$ y confirma su idoneidad en casos como los que presentamos en nuestro trabajo. El uso del fármaco es seguro y los efectos adversos infrecuentes en perfusiones controladas ${ }^{(8)}$, pero la práctica habitual hace que su manejo sea hospitalario, siendo en algunos casos como en nuestro centro, su uso supervisado por el Servicio de Anestesia y Reanimación.

La lidocaína por vía endovenosa presenta efectos adversos a nivel del Sistema Nervioso Central como convulsiones, efectos cardiovasculares (hipotensión severa, bradicardia y arritmias), mareo, somnolencia, agitación y llanto(9). Otros efectos adversos descritos con su infusión son: fotofobia, somnolencia, parestesia perioral, nausea, cefalea, disartria, xerostomía y sabor metálico. Nuestros pacientes únicamente presentaron somnolencia, que experimentaron previamente en su infusión 
hospitalaria; en ninguno de los casos interfirió posteriormente en el sueño nocturno ni en las actividades en el resto del día.

Se ha demostrado que las infusiones endovenosas de lidocaína administradas a dosis bajas de $1.5 \mathrm{mg} / \mathrm{kg}$ a 2-3 mg/min disminuye significativamente el dolor neuropático(7). La dosis correcta de lidocaína endovenosa para la resolución del dolor permanece en discusión ${ }^{(7)}$. Algunos estudios recogen control de dolor con dosis de $1 \mathrm{mg} / \mathrm{kg}$, y otros ensayos comparativos de 3 dosis de lidocaína endovenosa (1,3 y $5 \mathrm{mg} / \mathrm{kg}$ ) ${ }^{(7)}$, concluyen que la infusión de lidocaína a dosis de 1 y $3 \mathrm{mg} / \mathrm{kg} / \mathrm{h}$ no era mejor que placebo para resolver el dolor neuropático. Nuestros pacientes recibieron dosis de 3 $\mathrm{mg} / \mathrm{kg}$ con mejoría de las molestias como hemos indicado.

Desde hace años algunos sistemas de salud como el francés, tiene protocolizado el uso endovenoso de anestésicos locales en uso parenteral para uso paliativo ${ }^{(10)}$, una iniciativa que bien podríamos plantear en nuestro sistema. Consideramos la administración domiciliaria de lidocaína endovenosa paliativa como una alternativa eficaz y segura. Creemos que el uso del fármaco es seguro y supone una mejora asistencial para pacientes y familiares, por lo que debería considerarse su administración domiciliaria en condiciones de seguridad como como las descritas en este trabajo.

\section{BIBLIOGRAFÍA}

1. Regan JM, Peng P. Neurophisiology cancer pain. Cancer Control. 2000;7(2):111-9. DOI: 10.1177/107327480000700201

2. Stute P, Soukup J, Menzel M, Sabatowski R, Grond S. Analysis and treatment of different types of neuropathic cancer pain. J Pain Symtom Manage. 2003;26(6):1123-31. DOI: 10.1016/j.jpainsymman.2003.04.002

3. Tavares Mendonça F, Campos Reis M, Alvene Aguiar J, Calvano LA. Systemic lidocaine for perioperative analgesia: A literature review. J Anest Inten Care Med. 2015;1(1):e555551. DOI: 10.19080/JAICM.2015.01.555551

4. Scholz A. Mechanisms of (local) anaesthetics on voltage-gated sodium and other ion channels. Br J Anaesth. 2002;89(1):52-61. DOI: 10.1093/bja/aef163

5. Tanelian DL, Brose WG: Neuropathic pain can be relieved by drugs that are use-dependent sodium channel blockers: lidocaine, carbamazepine, and mexiletine. Anesthesiology. 1991;74(5):949-51. DOI: 10.1097/00000542-199105000-00026

6. Biella G, Sotgiu ML. Central effects of systemic lidocaine mediated by glycine spinal receptors: an iontophoretic study in the rat spinal cord. Brain Res. 1993;603(2):201-6. DOI: 10.1016/00068993(93)91238-n

7. Zhu B, Zhou X, Zhou Q, Wang H, Wang S, Luo K. Intra-Venous Lidocaine to Relieve Neuropathic Pain: A Systematic Review and Meta-Analysis. Front Neurol. 2019;10:954. DOI: 10.3389/ fneur.2019.00954.

8. Dunn LK, Durieux ME. Perioperative use of intravenous lidocaine. Anesthesiol. 2017;126(4):7297. DOI: $10.1097 / A L N .0000000000001527$

9. Carroll IR, Younger JW, Mackey SC. Pain quality predicts lidocaine analgesia among patients with suspected neuropathic pain. Pain Med. 2010;11(4):617-21. DOI: 10.1111/j.15264637.2010.00807.x

10. Salas S, Auquier P, Duffaud F, Garnier SR, Deschamps M, Honoré S, et al. Efficacy of lidocaine in patients receiving palliative care with opioid-refractory cancer pain with a neuropathic compo- 
Notas Clínicas: Villamil-Cajoto, Alvarez-Pérez, Marcos-Fernández et al

nent: study protocol for a randomized controlled study. Trials 2014;15:e318. DOI: 10.1186/17456215-15-318 